\title{
On some overdetermined free boundary problems
}

\author{
Samira Khatmi ${ }^{1} \quad$ Mohammed Barkatou ${ }^{2}$
}

(Received 23 October 2006; revised 19 November 2007)

\begin{abstract}
This article deals with some free boundary problems for the Laplacian operator. We first give sufficient conditions of existence of free boundaries. Then combining the maximum principle to the monotonicity of the mean curvature, we prove a symmetry result in the case where the source term is constant. The method presented in this work is new and all the results obtained here can be extended to more general divergence operators. The technique used to prove the symmetry result can be applied for other symmetric overdetermined value problems.
\end{abstract}

\section{Contents}

1 Introduction

See http://anziamj.austms.org.au/ojs/index.php/ANZIAMJ/article/view/168 for this article, (c) Austral. Mathematical Soc. 2007. Published November 22, 2007. ISSN 1446-8735 
2 Preliminaries

E14

3 Problem F(c,f)

E17

3.1 Shape optimization problems. . . . . . . . . . E E17

3.1.1 Existence of the minima . . . . . . . . . E18

3.1.2 The optimality conditions . . . . . . . . . . E19

3.1.3 Main theorems ............... E22

4 A symmetry result

E25

5 Concluding remarks

E28

References

E29

\section{Introduction}

Let $\mathrm{D}$ be an open and bounded subset of $\mathbb{R}^{N}(\mathrm{~N} \geq 2)$ which contains all the domains we use in this article. Let $c>0$ be a parameter and denote by $f$ a positive function. We look for an open set $\Omega \subset \mathrm{D}$ such that the following overdetermined free boundary problem has a solution.

$$
\mathrm{F}(\mathrm{c}, \mathrm{f})\left\{\begin{array}{lll}
-\Delta \mathrm{u}_{\Omega}=\mathrm{f} \quad \text { in } \Omega, & \mathrm{u}_{\Omega}=0 \text { on } \partial \Omega & \} \mathrm{P}(\Omega, f), \\
-\Delta v_{\Omega}=\mathrm{u}_{\Omega} \quad \text { in } \Omega, & v_{\Omega}=0 \text { on } \partial \Omega \quad & \mathrm{P}\left(\Omega, u_{\Omega}\right), \\
\frac{\partial u_{\Omega}}{\partial v} \times \frac{\partial v_{\Omega}}{\partial v}=\mathrm{c} \text { on } \partial \Omega & \text { (overdetermined condition). }
\end{array}\right.
$$

Here $v$ is the outward normal vector to $\partial \Omega$. Notice that since $u_{\Omega}=0=v_{\Omega}$ on $\partial \Omega$ then

$$
\left|\nabla u_{\Omega}\right|=-\frac{\partial u_{\Omega}}{\partial v} \quad \text { and } \quad\left|\nabla v_{\Omega}\right|=-\frac{\partial v_{\Omega}}{\partial v} .
$$

Therefore the overdetermined condition becomes

$$
\left|\nabla u_{\Omega}\right|\left|\nabla v_{\Omega}\right|=c \quad \text { on } \quad \partial \Omega .
$$


In general without any assumptions on data, the problem $F(c, f)$ has no solution.

Section 2 contains some preliminary results. In Section 3, we study the problem $F(c, f)$ when $f$ is a positive function belonging to $L^{2}(D)$ and having a compact support with nonempty interior: the solution we look for must strictly contains the convex hull of the support of $f$, say C. Our approach here consists of solving the shape optimization problem associated to $F(c, f)$. The boundary of the minimum $\Omega$ we obtain can touch $\partial C$. So, the shape derivative allows us to get the overdetermined condition only on $\partial \Omega \backslash C$. Our goal in this section is to give sufficient conditions in order to have $C$ strictly contained in $\Omega$ and $\left|\nabla \mathfrak{u}_{\Omega}\right|\left|\nabla v_{\Omega}\right|=\mathrm{c}$ on $\partial \Omega$, see Theorems 18 and 20. For Theorem 18 we apply the maximum principle, whereas for Theorem 20 we use the monotonicity of the mean curvature for the domains which are of class $C^{2}$. Section 4 concerns the case where $f \equiv 1$. This section proves that any solution of $F(c, 1)$ must be a ball. To get this symmetry result, we combine the maximum principle to the monotonicity of the mean curvature. Recall that when $f \equiv 2$, the problem $F(c, f)$ arises from a variational problem in Probability [11]. Kinateder and McDonald [11] used the first exit time for Brownian motion from a smoothly bounded domains in Euclidian space and defined two natural functionals on the space of embedded, compact, oriented, unparametrized hypersurfaces in Euclidian space. They developed explicit formulas for the first variation of the functionals and characterized the critical points as balls. Huang and Miller [10] established the variational formulas for maximizing the functionals (they considered) over $C^{k}$ domains with a volume constraint and obtained the same symmetry result for their maximizers. We present a new method to derive this symmetry result. We can also extend it to the p-Laplacian case (see Remark 26 at the end of Section 4). The last section concludes. 


\section{Preliminaries}

Definition 1 Let $\mathrm{K}_{1}$ and $\mathrm{K}_{2}$ be two compact subsets of $\mathrm{D}$. We call a Hausdorff distance of $\mathrm{K}_{1}$ and $\mathrm{K}_{2}$ (or briefly $\mathrm{d}_{\mathrm{H}}\left(\mathrm{K}_{1}, \mathrm{~K}_{2}\right)$ ) the following positive number:

$$
\mathrm{d}_{\mathrm{H}}\left(\mathrm{K}_{1}, \mathrm{~K}_{2}\right)=\max \left[\rho\left(\mathrm{K}_{1}, \mathrm{~K}_{2}\right), \rho\left(\mathrm{K}_{2}, \mathrm{~K}_{1}\right)\right] \text {, }
$$

where $\rho\left(K_{i}, K_{j}\right)=\max _{x \in K_{i}} d\left(x, K_{j}\right), i, j=1,2$, and $d\left(x, K_{j}\right)=\min _{y \in K_{j}}|x-y|$.

Definition 2 Let $\omega_{n}$ be a sequence of open subsets of $\mathrm{D}$ and $\omega$ be an open subset of $\mathrm{D}$. Let $\mathrm{K}_{\mathrm{n}}$ and $\mathrm{K}$ be their complements in $\overline{\mathrm{D}}$. We say that the sequence $\omega_{n}$ converges in the Hausdorff sense, to $\omega$ (or briefly $\omega_{n} \stackrel{H}{\longrightarrow} \omega$ ) if

$$
\lim _{n \rightarrow+\infty} d_{H}\left(K_{n}, K\right)=0
$$

Definition 3 Let $\omega_{n}$ be a sequence of open subsets of $\mathrm{D}$ and $\omega$ be an open subset of $\mathrm{D}$. We say that the sequence $\omega_{\mathrm{n}}$ converges in the compact sense to $\omega$ (or briefly $\omega_{\mathrm{n}} \stackrel{\mathrm{K}}{\longrightarrow} \omega$ ) if

- every compact subset of $\omega$ is included in $\omega_{\mathfrak{n}}$, for $\mathrm{n}$ large enough, and

- every compact subset of $\bar{\omega}^{\mathfrak{c}}$ is included in $\overline{\boldsymbol{\omega}}_{\mathfrak{n}}^{\mathfrak{c}}$, for $\mathrm{n}$ large enough.

Definition 4 Let $\omega_{n}$ be a sequence of open subsets of $\mathrm{D}$ and $\omega$ be an open subset of $\mathrm{D}$. We say that the sequence $\omega_{n}$ converges in the sense of characteristic functions, to $\omega$ (or briefly $\omega_{n} \stackrel{\mathrm{L}}{\longrightarrow} \omega$ ) if $\chi_{\omega_{n}}$ converges to $\chi_{\omega}$ in $\mathrm{L}_{l o c}^{\mathrm{p}}\left(\mathbb{R}^{\mathrm{N}}\right), \mathrm{p} \neq \infty,\left(\chi_{\omega}\right.$ is the characteristic function of $\left.\omega\right)$.

Lemma 5 [5, 15] If $\omega_{\mathrm{n}}$ is a sequence of open subsets of $\mathrm{D}$ then 
1. there exists a subsequence (still denoted by $\omega_{\mathrm{n}}$ ) which converges, in the Hausdorff sense, to some open subset of $\mathrm{D}$,

2. $|\partial \Omega| \leq \liminf _{n \rightarrow+\infty}\left|\partial \Omega_{n}\right|$.

Definition 6 [3] Let $\mathrm{C}$ be a compact convex set and let int(C) be its interior. The bounded domain $\mathrm{\omega}$ satisfies $\mathrm{C}$-GNP if

1. $\omega \supset \operatorname{int}(\mathrm{C})$,

2. $\partial \omega \backslash \mathrm{C}$ is locally Lipschitz,

3. for any $\mathrm{c} \in \partial \mathrm{C}$ there is an outward normal ray $\Delta_{\mathrm{c}}$ such that $\Delta_{\mathrm{c}} \cap \omega$ is connected, and

4. for every $\mathrm{x} \in \partial \mathrm{\omega} \backslash \mathrm{C}$ the inward normal ray to $\mathrm{\omega}$ (if exists) meets $\mathrm{C}$.

Put

$$
\mathcal{O}_{\mathrm{C}}=\{\omega \subset \mathrm{D}: \omega \text { satisfies } \mathrm{C} \text {-GNP }\}
$$

Theorem 7 If $\omega_{\mathrm{n}} \in \mathcal{O}_{\mathrm{C}}$, then there exists an open subset $\omega \subset \mathrm{D}$ and $a$ subsequence (again denoted by $\omega_{n}$ ) such that (i) $\omega_{n} \stackrel{\mathrm{H}}{\longrightarrow} \omega$, (ii) $\omega_{n} \stackrel{\mathrm{K}}{\longrightarrow} \omega$, (iii) $\chi_{\omega_{n}}$ converges to $\chi_{\omega}$ in $\mathrm{L}^{1}(\mathrm{D})$ and (iv) $\omega \in \mathcal{O}_{\mathrm{C}}$. Furthermore, the assertions (i), (ii) and (iii) are equivalent.

Barkatou proved this theorem [3, Theorem 3.1] and the equivalence between (i), (ii) and (iii) [3, Propositions 3.4, 3.5, 3.6, 3.7 and 3.8]. Notice that, in general, we do not have the equivalence between (i), (ii) and (iii) [9].

Proposition 8 Let $\left\{\omega_{n}, \omega\right\} \subset \mathcal{O}_{C}$ such that $\omega_{n} \stackrel{H}{\longrightarrow} \omega$. Let $\mathfrak{u}_{\mathfrak{n}}$ and $\mathfrak{u}_{\omega}$ be respectively the solutions of $\mathrm{P}\left(\omega_{n}, f\right)$ and $\mathrm{P}(\omega, \mathrm{w})$. Then $\boldsymbol{u}_{n}$ converges strongly in $\mathrm{H}_{0}^{1}(\mathrm{D})$ to $\mathrm{u}_{\omega}\left(\mathrm{u}_{\mathrm{n}}\right.$ and $\mathrm{u}_{\omega}$ are extended by zero in $\left.\mathrm{D}\right)$. 
This proposition was proven for $\mathrm{N}=2$ or 3 [3, Theorem 4.3].

Proposition 9 [4, Theorem 3.5] Let $v_{n}$ and $v_{\omega}$ be respectively the solutions of $\mathrm{P}\left(\omega_{n}, g_{n}\right)$ and $\mathrm{P}(\omega, \mathrm{g})$. If $\mathrm{g}_{\mathrm{n}}$ converges strongly in $\mathrm{H}^{-1}(\mathrm{D})$ to $\mathrm{g}$ then $v_{n}$ converges strongly in $\mathrm{H}_{0}^{1}(\mathrm{D})$ to $v_{\omega}\left(v_{n}\right.$ and $v_{\omega}$ are extended by zero in $\left.\mathrm{D}\right)$.

Definition 10 Let $\mathrm{C}$ be a convex set. We say that an open subset $\omega$ has the C-SP, if

\section{1. $\omega \supset \operatorname{int}(\mathrm{C})$,}

2. $\partial \omega \backslash \mathrm{C}$ is locally Lipschitz,

3. for any $\mathrm{c} \in \partial \mathrm{C}$ there is an outward normal ray $\Delta_{\mathrm{c}}$ such that $\Delta_{\mathrm{c}} \cap \omega$ is connected, and

4. for all $\mathrm{x} \in \partial \omega \backslash \mathrm{C} \quad \mathrm{K}_{\mathrm{x}} \cap \boldsymbol{\omega}=\emptyset$, where $\mathrm{K}_{\mathrm{x}}$ is the closed cone defined by

$$
\left\{y \in \mathbb{R}^{N}:(y-x) \cdot(z-x) \leq 0, \text { for all } z \in C\right\} .
$$

Remark $11 K_{x}$ is the normal cone to the convex hull of $C$ and $\{x\}$.

Proposition 12 [3, Proposition 2.3] $\omega$ has the C-GNP if and only if $\omega$ satisfies the $\mathrm{C}$-SP.

Theorem 13 [2, Theorem 1.4] Let $\mathrm{L}$ be a compact subset of $\mathbb{R}^{\mathrm{N}}$. Let $\mathrm{f}_{\mathrm{n}}$ be a sequence a functions defined on $\mathrm{L}$. We assume that the $\mathrm{f}_{\mathrm{n}}$ are of class $\mathrm{C}^{3}$ and

$$
\left|\frac{\partial f_{n}}{\partial x_{i}}\right| \leq M, \quad\left|\frac{\partial^{2} f_{n}}{\partial x_{i} \partial x_{j}}\right| \leq M, \quad\left|\frac{\partial^{3} f_{n}}{\partial x_{i} \partial x_{j} \partial x_{k}}\right| \leq M,
$$

where $\mathrm{M}$ is a strictly positive constant and is independent of $\mathrm{n}$. 
Define a sequence $\Omega_{\mathrm{n}}$, by $\Omega_{\mathrm{n}}=\left\{\mathrm{x} \in \mathrm{L}: \mathrm{f}_{\mathrm{n}}(\mathrm{x})>0\right\}$ and suppose there exists $\alpha>0$ such that $\left|\mathrm{f}_{\mathrm{n}}(\mathrm{x})\right|+\left|\nabla \mathrm{f}_{\mathrm{n}}(\mathrm{x})\right| \geq \alpha$ for all $\mathrm{x}$ in $\mathrm{L}$. If the $\Omega_{\mathrm{n}}$ have the $\mathrm{C}$-GNP, then there exists $\Omega$ of class $\mathrm{C}^{2}$ and a subsequence (still denoted by $\Omega_{\mathrm{n}}$ ) such that $\Omega_{\mathrm{n}}$ converges in the compact sense to $\Omega$.

Remark 14 The aim of Theorem 13 is to give the $C^{2}$ regularity of the minimum $\Omega$ (respectively $\Omega^{*}$ ) of $J$ (respectively $j$ ) defined below. This is in order to use the shape derivative and so to resolve Problem $F(c, f)$. The proof of this theorem uses the following lemma

Lemma 15 Let $\mathrm{L}$ be a compact subset of $\mathbb{R}^{\mathrm{N}}$. Let $\mathrm{f}_{\mathrm{n}}$ be a sequence of functions defined as Theorem 13. Suppose that $\Omega$ is an open subset of $\mathrm{L}$ such that

$$
\Omega=\{x \in L: h(x)>0\} \quad \text { and } \quad \partial \Omega=\{x \in L: h(x)=0\},
$$

where $\mathrm{h}$ is a continuous function defined in $\mathrm{L}$. If $\mathrm{f}_{\mathrm{n}}$ converges uniformly to $\mathrm{h}$ in $\mathrm{L}$, then the $\Omega_{\mathrm{n}}$ converge in the compact sense, to $\Omega$.

\section{Problem F(c,f)}

In this section, $N \in\{2,3\}$. If $\omega$ is an open subset of $D$, let $|\partial \omega|$ (respectively $|\omega|$ ) be the perimeter (respectively the volume) of $\omega$ and $H_{\partial \omega}$ be the mean curvature of $\partial \omega$.

\subsection{Shape optimization problems}

Consider the following shape optimization problem (OP).

Find $\Omega \in \mathcal{O}_{\mathrm{C}}$ such that $J(\Omega)=\min _{\omega \in \mathcal{O}_{\mathrm{C}}} J(\omega)$, 
where

$$
J(\omega)=c|\omega|-\frac{1}{2} \int_{\omega} u_{\omega}^{2} d x,
$$

with $|\boldsymbol{\omega}|$ the volume of $\boldsymbol{\omega}$ and $\mathfrak{u}_{\boldsymbol{\omega}}$ (respectively $\boldsymbol{v}_{\boldsymbol{\omega}}$ ) the solution of $\mathrm{P}(\boldsymbol{\omega}, \boldsymbol{f})$ (respectively $\mathrm{P}\left(\omega, \mathfrak{u}_{\omega}\right)$ ). Notice that by Green's formula,

$$
J(\omega)=c|\omega|-\frac{1}{2} \int_{\omega} f v_{\omega} d x
$$

\subsubsection{Existence of the minima}

Theorem 16 There exists $\Omega \in \mathcal{O}_{C}$ which is of class $C^{2}$ and minimizes the functional $\mathrm{J}$ on $\mathcal{O}_{\mathrm{C}}$. Furthermore, $\mathfrak{u}_{\Omega}$ (respectively $\boldsymbol{v}_{\Omega}$ ) is the solution of $\mathrm{P}(\Omega, \mathrm{f})$ (respectively $\mathrm{P}\left(\Omega, \mathrm{u}_{\Omega}\right)$ ).

Proof: Let $u_{D}$ be the solution of the Dirichlet problem $P(D, f)$, by the Maximum Principle, $0 \leq \mathfrak{u}_{\omega} \leq \mathfrak{u}_{D}$ so

$$
J(\omega)=c|\omega|-\frac{1}{2} \int_{\omega} u_{\omega}^{2} d x \geq-\frac{1}{2} \int_{D} u_{D}^{2},
$$

and inf J exists. Let $\Omega_{\mathfrak{n}}$ be a minimizing sequence in $\mathcal{O}_{C}$. We choose $\Omega_{n}$ as in Theorem 13 above and get the existence of a subsequence $\Omega_{n_{k}}$ and of $\Omega$ which is of class $C^{2}$ such that $\Omega_{n_{k}} \stackrel{K}{\longrightarrow} \Omega$. Then, from Theorem 7, (i) implies $\Omega_{n_{k}} \stackrel{H}{\longrightarrow} \Omega$, (iv) gives $\Omega \in \mathcal{O}_{C}$ and by (iii) $\left|\Omega_{n_{k}}\right|$ converges to $|\Omega|$. The convergence of $\int_{D} u_{n_{k}}^{2} \chi_{\Omega_{n_{k}}}$ to $\int_{D} u_{\Omega}^{2} \chi_{\Omega}$ is given by (iii) of Theorem 7 together with Proposition 8. We then conclude that $J(\Omega)=\min _{\boldsymbol{\omega} \in \mathcal{O}_{C}} J(\omega)$. Now, on one hand Proposition 8 implies that $u_{\Omega}$ is solution of $P(\Omega, f)$. On the other hand, Proposition 8 together with Proposition 9 implies that $v_{\Omega}$ is the solution of $\mathrm{P}\left(\Omega, \mathfrak{u}_{\Omega}\right)$.

Put

$$
\mathcal{O}_{\Omega}=\{\omega \subset \Omega: \omega \text { satisfies C-GNP }\}
$$


Consider

$$
j(\omega)=c|\partial \omega|+\int_{\partial \omega} \frac{\partial u_{\omega}}{\partial v} d x
$$

where $\mathfrak{u}_{\boldsymbol{\omega}}$ the solution of the Dirichlet problem $\mathrm{P}(\boldsymbol{\omega}, \mathrm{f})$. By Green's formula,

$$
j(\omega)=c|\partial \omega|-\int_{\omega} f(x) d x
$$

Theorem 17 There exists $\Omega^{*} \in \mathcal{O}_{\Omega}$ which minimizes the functional $\mathrm{j}$ on $\mathcal{O}_{\Omega}$. $\Omega^{*}$ is of class $\mathrm{C}^{2}$.

Proof: Since $f$ is positive, then for all $\omega \subset D$,

$$
j(\omega) \geq-\int_{D} f(x) d x .
$$

So inf $\boldsymbol{j}$ exists. As in the proof of Theorem 16 above, there exists a subsequence $\Omega_{\mathfrak{n}_{k}}^{*}$ and $\Omega^{*}$ which is of class $C^{2}$ such that $\Omega_{\mathfrak{n}_{k}}^{*} \stackrel{H}{\longrightarrow} \Omega$. Now, from Theorem 7, (iv) gives $\Omega^{*} \in \mathcal{O}_{\mathrm{C}}$ and by (iii) $\int_{\Omega_{n_{k}}^{*}} f(x) d x$ converges to $\int_{\Omega^{*}} f(x) d x$. Then item 2 of Lemma 5 permits to get $j\left(\Omega^{*}\right)=\min _{\omega \in \mathcal{O}_{C}} j(\omega)$.

\subsubsection{The optimality conditions}

In this subsection, we use the standard tool of the domain derivative to write down the optimality condition. Before doing this, recall the definition of the domain derivative $[17,14]$. Suppose that $\Omega$ is of class $C^{2}$. Consider a deformation field $V \in C^{2}\left(\mathbb{R}^{N} ; \mathbb{R}^{N}\right)$ and set $\Omega_{t}=\{x+t V(x): x \in \Omega\}$, $t>0$. The application $I d+t V$ (a perturbation of the identity) is a Lipschitz 
diffeomorphism for $t$ small enough and by definition, the derivative of $J$ at $\Omega$ in the direction $\mathrm{V}$ is

$$
\mathrm{dJ}(\Omega, V)=\lim _{\mathrm{t} \rightarrow 0} \frac{J\left(\Omega_{\mathrm{t}}\right)-J(\Omega)}{\mathrm{t}} .
$$

As the functional $J$ depends on the domain $\Omega$ through the solution of the Dirichlet problem $P(\Omega, f)$, we need to define also the domain derivative $u_{\Omega}^{\prime}$ of $u_{\Omega}$ :

$$
u_{\Omega}^{\prime}=\lim _{t \rightarrow 0} \frac{u_{\Omega_{t}}-u_{\Omega}}{t} .
$$

Furthermore, $\mathfrak{u}_{\Omega}^{\prime}$ is the solution of the following problem [17, 14]:

$$
\begin{cases}-\Delta u_{\Omega}^{\prime}=0 & \text { in } \Omega, \\ u_{\Omega}^{\prime}=-\frac{\partial u_{\Omega}}{\partial v} V \cdot v & \text { on } \partial \Omega,\end{cases}
$$

Now to compute the the shape derivatives of $\boldsymbol{J}$ and $\boldsymbol{j}$, recall the following [9].

1. The shape derivatives of the volume and the perimeter are respectively

$$
\int_{\partial \Omega} V \cdot v d \sigma \text { and } \int_{\partial \Omega} N_{\partial \Omega} V \cdot v d \sigma
$$

2. Since $u_{\Omega} \in H_{0}^{1}(D)$ and $\Omega$ is of class $C^{2}$, if we put

$$
F(\Omega)=\int_{\Omega} u_{\Omega}^{2} d x
$$

then the Hadamard formula gives

$$
\mathrm{dF}(\Omega, v)=2 \int_{\Omega} u_{\Omega} u_{\Omega}^{\prime} d x
$$

But $v_{\Omega}$ is solution to Dirichlet problem $\mathrm{P}\left(\Omega, \mathfrak{u}_{\Omega}\right)$, so by Green's formula

$$
\mathrm{dF}(\Omega, \mathrm{V})=2 \int_{\partial \Omega}\left|\nabla \mathrm{u}_{\Omega}\right|\left|\nabla v_{\Omega}\right| \mathrm{V} \cdot \boldsymbol{v} \mathrm{d} \sigma
$$


Now since $J(\omega)=c|\omega|-\frac{1}{2} F(\omega)$ then

$$
\mathrm{dJ}(\Omega, \mathrm{V})=\int_{\partial \Omega}\left(\mathrm{c}-\left|\nabla \mathrm{u}_{\Omega}\right|\left|\nabla v_{\Omega}\right|\right) \mathrm{V} \cdot \boldsymbol{v} \mathrm{d} \sigma .
$$

$\Omega$ being the minimum of the functional $\mathrm{J}, \mathrm{dJ}(\Omega, \mathrm{V}) \geq 0$ for every admissible direction $\mathrm{V}$. Therefore

$$
\int_{\partial \Omega}\left(\mathrm{c}-\left|\nabla \mathfrak{u}_{\Omega}\right|\left|\nabla v_{\Omega}\right|\right) \mathrm{V} \cdot \boldsymbol{v} \mathrm{d} \sigma \geq 0 \quad \text { for every admissible direction } \mathrm{V} \text {. }
$$

We mean by admissible direction the one which allows us to keep the C-GNP or the C-SP (according to Proposition 12). Since $\Omega$ has the C-GNP, it satisfies the C-SP. Then

$$
\text { for all } x \in \partial \Omega \backslash C, \quad K_{x} \cap \Omega=\emptyset .
$$

For $\mathrm{t}$ sufficiently small, let $\Omega_{\mathrm{t}}=\Omega+\mathrm{tV}(\Omega)$ be the deformation of $\Omega$ in the direction $V$. Let $x_{t} \in \partial \Omega_{t}$. There exists $x \in \partial \Omega$ such that $x_{t}=x+t V(x)$. Using the definition of $K_{x_{t}}$ and the equality above, we get (for $t$ small enough and for every displacement $\mathrm{V}$ )

$$
\text { for all } x_{t} \in \partial \Omega_{t} \backslash C, \quad K_{x_{t}} \cap \Omega_{t}=\emptyset,
$$

which means that $\Omega_{t}$ satisfies the C-SP (and so the C-GNP) for every direction $V$ when $t$ is sufficiently small. Then, using $V$ and $-V$, and that the set of the functions $V \cdot v$ is dense in $\mathrm{L}^{2}(\partial \Omega)$, we deduce

$$
\left|\nabla u_{\Omega}\right|\left|\nabla v_{\Omega}\right|=c \quad \text { on } \quad \partial \Omega \backslash \partial C .
$$

On the other hand, the admissible directions $V$ on $\partial \Omega \cap \partial C$ must satisfy

$$
V(x) \cdot v(x) \geq 0
$$

and one gets

$$
\left|\nabla \mathfrak{u}_{\Omega}\right|\left|\nabla v_{\Omega}\right| \leq \mathrm{c} \quad \text { on } \quad \partial \Omega \cap \partial \mathrm{C}
$$


Now, thanks to Hadamard formula, the shape derivative of $j$ at $\Omega^{*}$ is

$$
\operatorname{dj}\left(\Omega^{*}, \mathrm{~V}\right)=\int_{\partial \Omega^{*}}\left(\mathrm{NcH}_{\partial \Omega^{*}}-\mathrm{f}\right) \mathrm{V} \cdot v \mathrm{~d} \sigma \geq 0
$$

for every admissible direction $\mathrm{V}$.

Arguing as above and observing that the deformation domain $\Omega_{\mathrm{t}}^{*}$ is in $\Omega$ if the directions $V$ on $\partial \Omega^{*} \cap \partial \Omega$ satisfy $V(x) \cdot v(x) \leq 0$, we get

$$
\begin{cases}\mathrm{H}_{\partial \Omega^{*}}=0 & \text { on } \partial \Omega^{*} \backslash(\partial \mathrm{C} \cup \partial \Omega), \\ \mathrm{H}_{\partial \Omega^{*}} \leq 0 & \text { on } \partial \Omega^{*} \cap \partial \Omega, \quad \text { and } \\ \mathrm{H}_{\partial \Omega^{*}} \geq \frac{f}{N c} & \text { on } \partial \Omega^{*} \cap \partial \mathrm{C} .\end{cases}
$$

\subsubsection{Main theorems}

Theorem 18 Let $\Omega$ be as in Theorems 16 and $\mathrm{C}$ be of class $\mathrm{C}^{2}$. Let $\mathfrak{u}_{\mathrm{C}}$ $\left(\right.$ respectively $\left.v_{\mathrm{C}}\right)$ be the solution of $P(\operatorname{int}(\mathrm{C}), \mathrm{f})\left(\right.$ respectively $\left.P\left(\operatorname{int}(\mathrm{C}), \mathrm{u}_{\mathrm{C}}\right)\right)$. Suppose that both $\mathrm{u}_{\mathrm{C}}$ and $v_{\mathrm{C}}$ are in $\mathrm{C}^{2}(\mathrm{C})$ and

$$
\left|\nabla u_{\mathrm{C}}\right| \nabla v_{\mathrm{C}} \mid>\mathrm{c} \quad \text { on } \quad \partial \mathrm{C} \text {. }
$$

Then $\Omega$ is a solution of $F(\mathbf{c}, \mathbf{f})$ which strictly contains $C$.

Proof: $\partial \Omega \neq \partial C$, otherwise $\Omega=\operatorname{int}(C), u_{\Omega}=u_{C}$ and $v_{\Omega}=v_{C}$. But the optimality conditions (3) and (4) give

$$
\left|\nabla \mathfrak{u}_{\mathrm{C}}\right|\left|\nabla v_{\mathrm{C}}\right|=\left|\nabla \mathfrak{u}_{\Omega}\right|\left|\nabla v_{\Omega}\right| \leq \mathrm{c} \quad \text { on } \partial \mathrm{C},
$$

which contradicts (6).

Now, suppose that $\partial \Omega \cap \partial C \neq \emptyset$. Since $\boldsymbol{u}_{\Omega}$ and $\boldsymbol{u}_{C}$ are in $C^{2}(C)$,

$$
\Delta u_{\Omega}=-f=\Delta u_{C} \quad \text { in } \operatorname{int}(C) \text { and } u_{\Omega} \geq 0=u_{C} \quad \text { on } \partial C,
$$


the maximum principle implies that

$$
u_{\Omega} \geq u_{C} \quad \operatorname{in} \operatorname{int}(C) .
$$

But $\mathfrak{u}_{\Omega} \neq \mathfrak{u}_{\mathrm{C}}$ in $\operatorname{int}(\mathrm{C})$, then

$$
u_{\Omega}>u_{C} \quad \operatorname{in} \operatorname{int}(C) .
$$

Now, since $\mathfrak{u}_{\Omega}=\mathfrak{u}_{\mathrm{C}}$ on $\partial \Omega \cap \partial \mathrm{C}$, then by the maximum principle we get

$$
\frac{\partial u_{\Omega}}{\partial v}<\frac{\partial u_{C}}{\partial v} \quad \text { on } \partial \Omega \cap \partial C
$$

or again, since $\left|\nabla u_{\Omega}\right|=-\frac{\partial u_{\Omega}}{\partial v}$,

$$
\left|\nabla \mathfrak{u}_{\mathrm{C}}\right|<\left|\nabla \mathfrak{u}_{\Omega}\right| \quad \text { on } \partial \Omega \cap \partial \mathrm{C} .
$$

Now by the maximum principle, $\mathfrak{u}_{\Omega} \geq 0$ on $\bar{\Omega}$. Then replacing $f$ by $\mathfrak{u}_{\Omega}, \mathfrak{u}_{\Omega}$ by $v_{\Omega}$ and $u_{\mathrm{C}}$ by $v_{\mathrm{C}}$, and arguing as above, we obtain

$$
\left|\nabla v_{\mathrm{C}}\right|<\left|\nabla v_{\Omega}\right| \quad \text { on } \partial \Omega \cap \partial \mathrm{C} .
$$

But (6) together with (4) implies

$$
\mathrm{c}<\left|\nabla \mathrm{u}_{\mathrm{C}}\right|\left|\nabla \mathrm{v}_{\mathrm{C}}\right|<\left|\nabla \mathrm{u}_{\Omega}\right|\left|\nabla v_{\Omega}\right| \leq \mathrm{c} \quad \text { on } \partial \mathrm{C} \cap \partial \Omega .
$$

which is absurd. It then follows that $C$ is strictly contained in $\Omega$ and so

$$
\left|\nabla u_{\Omega}\right|\left|\nabla v_{\Omega}\right|=c \quad \text { on } \partial \Omega
$$

Theorem 19 Let $\Omega$ and $\Omega^{*}$ be as in Theorems 16 and 17. Suppose

$$
\left|\nabla u_{\Omega^{*}}\right|\left|\nabla v_{\Omega^{*}}\right|>c \quad \text { on } \partial \Omega^{*} .
$$

Then $\Omega$ is a solution of $F(c, f)$ which strictly contains $\Omega^{*}$. 
Replacing $\Omega$ by $\Omega^{*}$, the proof is the same as above.

Theorem 20 Let $\Omega$ and $\Omega^{*}$ be as in Theorems 16 and 1\%. Suppose that $\mathrm{C}$ is of class $\mathrm{C}^{2}$ and

$$
\mathrm{H}_{\partial \mathrm{C}}<\frac{\mathrm{f}}{\mathrm{Nc}} \quad \text { on } \partial \mathrm{C} \text {. }
$$

Then

1. C is strictly contained in $\Omega^{*}$,

2. $\Omega$ is a solution of $F(\mathrm{c}, \mathrm{f})$ which contains $\Omega^{*}$.

\section{Proof:}

1. Suppose by contradiction that $\partial \Omega^{*} \cap \partial C \neq \emptyset$. Since int $(C) \subset \Omega^{*}$ and $C$ and $\Omega^{*}$ are of class $C^{2}$, then by using the monotonicity of the mean curvature together with (5) and (8) we have on $\partial \Omega^{*} \cap \partial C$

$$
\frac{f}{N c} \leq H_{\partial \Omega^{*}} \leq H_{\partial c}<\frac{f}{N c},
$$

which gives a contradiction.

2. $\mathrm{C}$ is strictly contained in $\Omega^{*}$ which is contained in $\Omega$ (by definition). So C is strictly contained in $\Omega$ and (3) gives the overdetermined condition on $\partial \Omega$. 


\section{A symmetry result}

In this section, we suppose that $f \equiv 1$ and $N \geq 2$.

Definition 21 [6] We say that a domain $\omega$ satisfies the $\varepsilon$-cone property if for all $\chi \in \partial \omega$ there exist a direction vector $\xi \in \mathbb{R}^{N}$ such that the cone $\mathrm{C}(\mathrm{y}, \xi, \varepsilon) \subset \omega$ for all $\mathrm{y} \in \mathrm{B}(\mathrm{x}, \varepsilon) \cap \bar{\omega}$. $\varepsilon$ denotes both angle and hight of the cone.

Denoting by $\mathcal{O}_{\varepsilon}$ the class of domains which have the $\mathcal{\varepsilon}$-cone property, we have this lemma.

Lemma 22 [6] If $\omega_{\mathrm{n}} \in \mathcal{O}_{\varepsilon}$, then there exists an open subset $\omega \subset \mathrm{D}$ and $a$ subsequence (again denoted by $\omega_{n}$ ) such that (i) $\omega_{n} \stackrel{H}{\longrightarrow} \omega$, (ii) $\bar{\omega}_{n} \stackrel{H}{\longrightarrow} \bar{\omega}$, (iii) $\partial \omega_{n} \stackrel{H}{\longrightarrow} \partial \omega$, (iv) $\chi_{\omega_{n}}$ converges to $\chi_{\omega}$ in $\mathrm{L}^{1}(\mathrm{D})$ and $(v) \omega \in \mathcal{O}_{\varepsilon}$, (vi) $\nu_{\omega_{n}}$ converges strongly in $\mathrm{H}_{0}^{1}(\mathrm{D})$ to $\nu_{\omega}\left(v_{\omega_{n}}\right.$ and $v_{\omega}$ are respectively the solution of $\mathrm{P}\left(\omega_{n}, 1\right)$ and $\left.\mathrm{P}(\omega, 1)\right)$.

Let $\Omega$ be a solution of the problem $F(c, 1)$ and put

$$
\mathcal{O}_{1}=\left\{\omega \supset \Omega: \omega \in \mathcal{O}_{\varepsilon}\right\} \text {. }
$$

Consider

$$
\mathrm{J}_{1}(\omega)=\mathrm{c}^{2}|\partial \omega|-\frac{1}{2} \int_{\omega} \mathrm{u}_{\omega}^{2}
$$

where $u_{\omega}$ is the solution of the Dirichlet problem $P(\omega, 1)$.

Before stating the main theorem of this section, let us state the two following propositions. The proofs are analogous to those of Theorems 16 and 17 . They use Lemma 22 together with the lower semicontinuity of the perimeter and the continuity of the volume (for the Hausdorff topology). To obtain 
the optimality conditions, we use the shape derivative as in Subsection 3.1.2. Using the same notations, to get $\Omega$ in $\left(\Omega_{1}\right)_{t}$ (for $t$ small enough) we must choose a deformation directions $V$ such that $V \cdot v \geq 0$ on $\partial \Omega \cap \partial \Omega_{1}$. In the same way $V \cdot v \leq 0$ on $\partial \Omega \cap \partial \Omega_{2}$ allows us to have $\left(\Omega_{2}\right)_{\mathrm{t}}$ in $\Omega\left(\Omega_{1}\right.$ and $\Omega_{2}$ are given by Propositions 23 and 24).

Proposition 23 There exists $\Omega_{1} \in \mathcal{O}_{1}$ which minimizes $\mathrm{J}_{1}$ on $\mathcal{O}_{1}$. If $\Omega_{1}$ is of class $\mathrm{C}^{2}$, then
1. $\left|\nabla u_{\Omega_{1}}\right|\left|\nabla v_{\Omega_{1}}\right| \leq \mathrm{Nc}^{2} \mathrm{H}_{\partial \Omega_{1}}$ on $\partial \Omega_{1} \cap \partial \Omega$,
2. $\left|\nabla u_{\Omega_{1}}\right|\left|\nabla v_{\Omega_{1}}\right|=\mathrm{Nc}^{2} \mathrm{H}_{\partial \Omega_{1}}$ on $\partial \Omega_{1} \backslash \partial \Omega$.

Consider

$$
\mathcal{O}_{2}=\left\{\omega \subset \Omega: \partial \omega \cap \partial \Omega_{1} \neq \emptyset \text { and } \omega \in \mathcal{O}_{\varepsilon}\right\}
$$

and

$$
\mathrm{J}_{2}(\omega)=\mathrm{c}|\partial \omega|-|\omega|
$$

Proposition 24 There exists $\Omega_{2} \in \mathcal{O}_{2}$ which minimizes $\mathrm{J}_{2}$ on $\mathcal{O}_{2}$. If $\Omega_{2}$ is of class $\mathrm{C}^{2}$, then
1. $H_{\partial \Omega_{2}} \leq \frac{1}{N_{c}}$ on $\partial \Omega \cap \partial \Omega_{2}$,
2. $\mathrm{H}_{\partial \Omega_{2}}=\frac{1}{\mathrm{Nc}}$ on $\partial \Omega_{2} \backslash \partial \Omega$.

In the sequel, we state and prove the main theorem of this section.

Theorem 25 If $\Omega$ is a solution of $F(\mathrm{c}, 1)$, then $\Omega$ is a ball of radius $\mathrm{Nc}$.

As we see in the following proof, to reach the conclusion of the theorem, we show that $\Omega=\Omega_{1}$. 
Proof: Since $\Omega \subset \Omega_{1}$, it is sufficient to show that $\partial \Omega=\partial \Omega_{1}$. By Lemma 22 and the definition of $\mathcal{O}_{2}, \partial \Omega_{2} \cap \partial \Omega_{1} \neq \emptyset$. So since $\Omega_{2} \subset \Omega \subset \Omega_{1}$ then

$$
\partial \Omega_{2} \cap \partial \Omega \cap \partial \Omega_{1} \neq \emptyset .
$$

This together with the monotonicity of the mean curvature and the fact that $\Omega, \Omega_{1}$ and $\Omega_{2}$ are of class $C^{2}$, implies that for all $x \in \partial \Omega_{2} \cap \partial \Omega \cap \partial \Omega_{1}$,

$$
H_{\partial \Omega_{1}}(x) \leq H_{\partial \Omega}(x) \leq H_{\partial \Omega_{2}}(x) \leq \frac{1}{N c} .
$$

Hence, Propositions 23 and 24 imply

$$
\left|\nabla u_{\Omega_{1}}(x)\right|\left|\nabla v_{\Omega_{1}}(x)\right| \leq \mathrm{Nc}^{2} \mathrm{H}_{\partial \Omega_{2}}(x) \leq \mathrm{c} .
$$

Suppose that $\partial \Omega \neq \partial \Omega_{1}$, by the maximum principle we obtain

$$
\mathrm{c}=\left|\nabla \mathfrak{u}_{\Omega}(\mathrm{x})\right|\left|\nabla v_{\Omega}(\mathrm{x})\right|<\left|\nabla \mathfrak{u}_{\Omega_{1}}(\mathrm{x})\right|\left|\nabla v_{\Omega_{1}}(\mathrm{x})\right| \leq \mathrm{c},
$$

which is a contradiction. It then follows that $\Omega=\Omega_{1}$ and so

$$
c=\left|\nabla u_{\Omega}\right|\left|\nabla v_{\Omega}\right|=\mathrm{Nc}^{2} \mathrm{H}_{\partial \Omega} \quad \text { on } \partial \Omega .
$$

This implies that

$$
\mathrm{H}_{\partial \Omega}=\frac{1}{\mathrm{Nc}}
$$

which means that $\Omega$ is a ball of radius $N c$ thanks to Alexandrov's result [1]. In this case, (9) implies $\Omega_{2}=\Omega$.

Remark 26 Combining the Hopf's comparison principle [18] with the monotonicity of the mean curvature, one can extend the result of Theorem 25 to the case of the p-Laplace operator. 


\section{Concluding remarks}

Remark 27 Recall that the C-GNP is satisfied by the solution of the socalled Quadrature Surface Free Boundary Problem [16, 8]. The domain which satisfies the C-GNP may be non-Lipschitz, it may contain cusps [3]. As a consequence, $\mathcal{O}_{\mathrm{C}}$ cannot be contained in $\mathcal{O}_{\varepsilon}$.

Remark 28 The hypothesis in Theorem 13 about the local regularity is not too restrictive because of, for instance, results due to DiBenditto [7], Lewis [12] and Lieberman [13].

Remark 29 For the p-Laplacian, the continuity with respect to the domain is a consequence of the $\gamma_{p}$-convergence [4]. So using Hopf's comparison principle and replacing the condition (6) by

$$
\left|\nabla u_{\mathrm{C}}\right|\left|\nabla v_{\mathrm{C}}\right|>\mathrm{c} \quad \text { on } \mathrm{C} .
$$

one can extend Theorems 18 and 19 to the p-Laplacian case.

Remark 30 Theorem 20 can be extended to other divergence operators like $\operatorname{div}(a(x, D u))$. For this kind of operators the continuity with respect to the domain is a simple consequence of Mosco convergence [4].

Remark 31 Consider the following free boundary problem. We look for a domain $\Omega$ and a couple of functions $\left(u_{\Omega} ; v_{\Omega}\right)$ such that $u_{\Omega}$ is solution of $\mathrm{P}(\Omega, f), v_{\Omega}$ is solution to $\mathrm{P}\left(\Omega, \mathfrak{u}_{\Omega}\right)$ and

$$
\left|\nabla u_{\Omega}\right|\left|\nabla v_{\Omega}\right|=\sigma \mathrm{H}_{\partial \Omega}+\mathrm{c} \quad \text { on } \partial \Omega .
$$

By considering the functional of domain

$$
J_{\sigma}(\omega)=\frac{\sigma}{N}|\partial \omega|+c|\omega|-\frac{1}{2} \int_{\omega} u_{\omega}^{2} d x
$$


we use the same arguments as in Theorem 16 and prove the existence of a minimum $\Omega$ for $\mathrm{J}_{\sigma}$ on $\mathcal{O}_{\mathrm{C}}$ which is of class $\mathrm{C}^{2}$. Then performing the shape derivative we obtain the following optimality conditions

$$
\begin{array}{ll}
\left|\nabla u_{\Omega}\right|\left|\nabla v_{\Omega}\right|=\sigma \mathrm{H}_{\partial \mathrm{c}}+\mathrm{c} & \text { on } \partial \Omega \backslash \partial \mathrm{C} \\
\left|\nabla \mathrm{u}_{\Omega}\right|\left|\nabla v_{\Omega}\right| \leq \sigma \mathrm{H}_{\partial \mathrm{C}}+\mathrm{c} & \text { on } \partial \Omega \cap \partial \mathrm{C} .
\end{array}
$$

Next, replacing in the inequality (6) of Theorem 18, c by $\sigma \mathrm{H}_{\partial \mathrm{c}}+\mathrm{c}$ we obtain the same conclusion by combining the maximum principle with the monotonicity of the mean curvature. Now, according to Remark 30 we can extend this result to the p-Laplacian case.

\section{References}

[1] A. D. Alexandrov: Uniquness theorems for surfaces in large I, II, Amer. Soc. Trans. 31, 1962, 341-388. E27

[2] M. Barkatou, D. Seck and I. Ly: An existence result for a quadrature surface free boundary problem, Cent. Eur. Jou. Math. 3(1), 2005, 39-57. E16

[3] M. Barkatou: Some geometric properties for a class of non Lipschitz-domains, New York J. Math. 8, 2002, 189-213. http://nyjm.albany.edu:8000/j/2002/8-13.html E15, E16, E28

[4] D. Bucur and P. Trebeschi : Shape Optimization Problems Governed by Nonlinear State Equations, Proc. Roy. Soc. Edinburgh, 128A, 1998, 945-963 E16, E28

[5] D. Bucur and J.P. Zolesio : N-dimensional shape optimization under capacitary constraints, J. Diff. Eq., 123-2, 1995, 504-522. E14 
[6] D. Chenais : Sur une famille de variétés à bord lipschitziennes, application à un problème d'identification de domaine, Ann. Inst. Fourier, 4-27, 1977, 201-231. E25

[7] E. DiBenditto : $\mathrm{C}^{1+\alpha}$ local regularity of weak solutions of degenerate elliptic equations, Nonlinear Analysis, 7, 1983, 827-850. E28

[8] B. Gustafsson and H. Shahgholian : Existence and geometric properties of solutions of a free boundary problem in potential theory, J. für die Reine und Ang. Math. 473, 1996, 137-179. E28

[9] A. Henrot and M. Pierre : Variation et optimisation de formes, une analyse geometrique, Mathmatiques and Applications, 48, (2005), SMAI Springer E15, E20

[10] C. Huang and D. Miller : Domain functionals and exit times for Brownian motion. Proc. Amer. Math. Soc. 130(3), 2001, 825-831. E13

[11] K. K. J. Kinateder P. Mcdonald : Hypersurfaces in $\mathrm{R}^{\mathrm{d}}$ and the variance of times for Brownian motion. Proc. Amer. Math. Soc. 125(8), 1997, 2453-2462. E13

[12] J. L. Lewis : Regularity of the derivatives of solutions to certain degenerate elliptic equations, Indiana Univ. Math. J., 32, 1983, 849-858. E28

[13] G. M. Liberman : Boundary regularity for solutions of degenerate elliptic equations, Nonlinear Analysis, 12, 1988, 1203-1219. E28

[14] F. Murat and J. Simon: Quelques résultats sur le contrôle par un domaine géométrique, Publ. du labo. d'Anal. Num. Paris VI, 1974, 1-46 E19, E20

[15] O. Pironneau : Optimal shape design for elliptic systems, Springer Series in Computational Physics, 1984, Springer, New York. E14 
[16] H. Shahgholian : Existence of Quadrature Surfaces for Positive Measures with Finite Support, Potential Analysis, 3, 1994, 245-255. E28

[17] J. Sokolowski et J. P. Zolesio : Introduction to shape optimization : shape sensitity analysis, Springer Series in Computational Mathematics, 10, 1992, Springer, Berlin. E19, E20

[18] P. Tolksdorf (1983) On the Dirichlet problem for quasilinear equations in domains with conical boundary points, Comm. Partial Differential Equations, 8(7), 1983, 773-817. E27 


\section{Author addresses}

\section{Samira Khatmi,}

mailto:khatmi@hotmail.com

2. Mohammed Barkatou, University Chouaib Doukkali mailto:mbarkatou@hotmail.com 